\title{
TXNRD1 Is an Unfavorable Prognostic Factor for Patients with Hepatocellular Carcinoma
}

\author{
Binsheng Fu, ${ }^{1}$ Wei Meng, ${ }^{1}$ Xiancheng Zeng, ${ }^{2}$ Hui Zhao, ${ }^{1}$ Wei Liu, ${ }^{3}$ and Tong Zhang ${ }^{1}$ \\ ${ }^{1}$ Department of Hepatic Surgery and Liver Transplantation Center of the Third Affiliated Hospital, \\ Organ Transplantation Institute of Sun Yat-sen University, Organ Transplantation Research Center of Guangdong Province, \\ Guangzhou 510630, China \\ ${ }^{2}$ Department of General Surgery, The Second People's Hospital of Guangdong Province, Guangzhou 510317, China \\ ${ }^{3}$ Guangdong Provincial Key Laboratory of Liver Disease Research and Key Laboratory of \\ Clinical \& Translational Research on Biotherapy for Liver Disease of Guangdong Higher Education Institutes and \\ Cell-Gene Therapy Translational Medicine Research Center of The Third Affiliated Hospital, Sun Yat-sen University, \\ Guangzhou, Guangdong 510630, China \\ Correspondence should be addressed to Wei Liu; uv1@163.com and Tong Zhang; zhjeff72@sina.com
}

Received 5 December 2016; Revised 6 February 2017; Accepted 12 February 2017; Published 27 April 2017

Academic Editor: Noriyoshi Sawabata

Copyright (C) 2017 Binsheng Fu et al. This is an open access article distributed under the Creative Commons Attribution License, which permits unrestricted use, distribution, and reproduction in any medium, provided the original work is properly cited.

\begin{abstract}
Thioredoxin reductase 1 (TXNRD1) which is a selenocysteine-containing protein is overexpressed in many malignancies. Its role in the hepatocellular carcinoma (HCC) prognosis has not been investigated. In this study, we investigated whether TXNRD1 functions as an independent prognostic factor for HCC patients. We found TXNRD1 was overexpressed in HCC tissues and cells, immunohistochemical analysis suggested TXNRD1 was elevated in 57 of 120 (47.5\%) clinical samples, and its level was increased with the increasing clinical stage. In addition, TXNRD1 expression was positively correlated with clinical stage $(p=3.5 e-5)$, N classification $(p=4.4 e-4)$, and M classification $(p=0.037)$ of HCC patients. Kaplan-Meier analysis revealed that patients with high TXNRD1 expression had significantly shorter survival time than patients with low TXNRD1 expression. Multivariate analysis found TXNRD1 was an independent prognostic factor for HCC patients. In conclusion, our data suggested that TXNRD1 was a biomarker for the prognosis of patients with HCC.
\end{abstract}

\section{Introduction}

HCC is one of the most common human tumors worldwide. According to the data published by the World Cancer Report 2014 , the global incidence and mortality of HCC are $6 \%$ and $9 \%$, respectively. About 746,000 patients died at 2012. Despite the advances in diagnosis and therapy, HCC still has poor prognosis and high recurrence rate, and looking for new diagnostic factors and therapeutic factors is critical for HCC therapy [1].

Selenoprotein TXNRD1 is a critical antioxidant enzyme catalyzing the NADPH-dependent reduction of thioredoxin to regulate cellular redox homeostasis [2]. It associated with cell proliferation, apoptosis, and transformation [3]. TXNRD1 is also upregulated in many human malignancies and functions as a prognostic factor for many tumors, such as oral squamous cell carcinomas [4], lung cancer $[5,6]$, breast cancer [7, 8], and astrocytomas [9]. Previous reports have shown that the hepatocarcinogenesis rate in TXNRD1 knockout mice was significantly increased, induced by carcinogen diethylnitrosamine (DEN), suggesting it could protect against chemically induced hepatocarcinogenesis through controlling the balance of cellular redox state, but it could not induce hepatocarcinogenesis [10]. Anticancer natural product gambogic acid interacts with TXNRD1 to inhibit its Trx-reduction activity and to increase the levels of reactive oxygen species to induce apoptosis in human HCC cell SMMC-7721; meanwhile, knockdown of TXNRD1 attenuates the gambogic acid cytotoxicity [11]. These findings suggested TXNRD1 might regulate the progression of HCC, but whether TXNRD1 could serve as a novel prognostic factor for HCC patients has not been studied until now. In this study, 
TABLE 1: Clinicopathological characteristics of HCC patients' samples.

\begin{tabular}{lc}
\hline & Number of cases $(\%)$ \\
\hline Gender & \\
Male & $88(73.3 \%)$ \\
Female & $32(26.7 \%)$ \\
Age (years) & \\
$\geq 45$ & $76(63.3 \%)$ \\
$<45$ & $44(36.7 \%)$ \\
Clinical stage & \\
I & $30(25.0 \%)$ \\
II & $30(25.0 \%)$ \\
III & $34(28.3 \%)$ \\
IV & $26(21.7 \%)$ \\
T classification & \\
T1 & $39(32.5 \%)$ \\
T2 & $33(27.5 \%)$ \\
T3 & $18(15.0 \%)$ \\
T4 & $30(25.0 \%)$ \\
N classification & \\
N0 & $98(81.7 \%)$ \\
N1 & $22(18.3 \%)$ \\
M classification & \\
Yes & $7(5.8 \%)$ \\
No & $113(94.2 \%)$ \\
\hline
\end{tabular}

we determined the role of TXNRD1 in the prognosis for HCC patients, and we showed that TXNRD1 was upregulated in HCC cells and tissues and was an unfavorable prognostic factor for HCC patients.

\section{Materials and Methods}

2.1. Cell Culture and Clinical Samples. Hepatocyte cell line LO2 cells were grown in RPMI-1640 medium (Gibco) supplemented with $10 \%$ fetal bovine serum (FBS, Gibco), $0.3 \%$ glutamine, $0.1 \mathrm{mM}$ nonessential amino acids, $1 \mathrm{mM}$ sodium pyruvate, and $10 \mu \mathrm{M} \beta$-mercaptoethanol (Gibco). HCC cell lines including Hep3B, BEL-7404, HepG2, Huh7, QGY-7703, MHCC97H, MHCC97L, BEL-7402, and HCCC-9801 were maintained in DMEM medium (Hyclone) supplemented with 10\% FBS (Gibco). These cells were obtained from ATCC.

A cohort of 120 paraffin-embedded HCC samples was obtained from the Third Affiliated Hospital of Sun Yatsen University (Guangzhou, China) with informed consent according to the Institute Research Ethics Committee; these patients were histologically and clinically diagnosed between July 2008 and November 2014. The detailed information was shown in Table 1, clinicopathological characteristics such as gender, age, clinical stage, and TNM classification were included.

2.2. Western Blot. Cell lysates were extracted from cells using RIPA buffer (50 mM Tris ( $\mathrm{pH} 7.4$ ), $150 \mathrm{mM} \mathrm{NaCl}$, $1 \%$ Triton $\mathrm{X}-100,1 \%$ sodium deoxycholate, and $0.1 \%$ SDS supplemented with protease inhibitor cocktail (Roche)). Protein concentration was measured using BCA Protein Assay Kit (Pierce). Equivalent protein lysates were separated on $12 \%$ SDS-PAGE gels and transferred to PVDG membranes (Millipore) followed with blocking with 5\% nonfat milk for $1 \mathrm{~h}$ at room temperature. After blocking, membranes were incubated with anti-TXNRD1 antibodies (1:1000, \#15140, Cell Signaling) for $2 \mathrm{~h}$ at room temperature; then, the membranes were washed using TBST buffer and incubated with an anti-rabbit IgG, HRP-linked antibody $(1: 10000$, \#7074, Cell Signaling) for $1 \mathrm{~h}$ at room temperature. The band was detected using Amersham ECL Prime (GE Healthcare). The membranes were stripped and reprobed with an anti- $\beta$-actin antibody as the loading control.

2.3. Immunohistochemistry (IHC). IHC was performed according to the standard method described previously $[12,13]$. Two observers who were blinded to the clinical outcome evaluated and scored the degree of immunostaining of TXNRD1 independently. The staining index was calculated as the sum of the intensity of staining and the percentage of positively stained tumor cells. Scores for the intensity of staining were shown as follows: 0 (no staining), 1 (weak staining, light yellow), 2 (moderate staining, yellow brown), and 3 (strong staining, brown). Scores for the percentage of positively stained tumor cells were shown as follows: 0 (no positive tumor cells), 1 ( $<10 \%$ of the cells), 2 (10-50\% of the cells), 3 ( $>50 \%$ of the cells). The staining index scores were 0 , $1,2,3,4,6$ and 9. The cut-off values for TXNRD1 expression were chosen based on the measure of heterogeneity using the log-rank test with respect to the overall survival. When SI score was $\geq 4$, the tumor was considered to have high expression; otherwise, the tumor was considered to have low expression.

2.4. Statistical Analysis. All statistical analyses in this study were carried out using SPSS 13.0 (IBM). Chi-square test and Fisher's test were used to determine the relationship between TXNRD1 expression and the clinicopathological characteristics, and Spearman correlation test was performed to calculate bivariate correlations between the study variables. The survival curves were plotted by the Kaplan-Meier method and compared by log-rank test. Univariate and multivariate analysis was carried out using Cox's proportional hazards regression models. For all tests, a two-sided $p$ value of less than 0.05 was considered to be statistically significant.

\section{Results}

3.1. TXNRD1 Is Overexpressed in HCC Tissues and Cells. To investigate the prognostic value of TXNRD1, we first examined TXNRD1 expression in HCC tissues. We found TXNRD1 mRNA level was significantly upregulated in HCC tissues compared to liver nontumor tissues ( $p<0.0001)$, mRNA expression profiles of HCC and liver nontumor tissues came from GSE14520 (Figure 1(a)), we also used The Cancer Genome Atlas (TCGA) dataset to analyze TXNRD1 expression, and TXNRD1 mRNA level was also significantly 


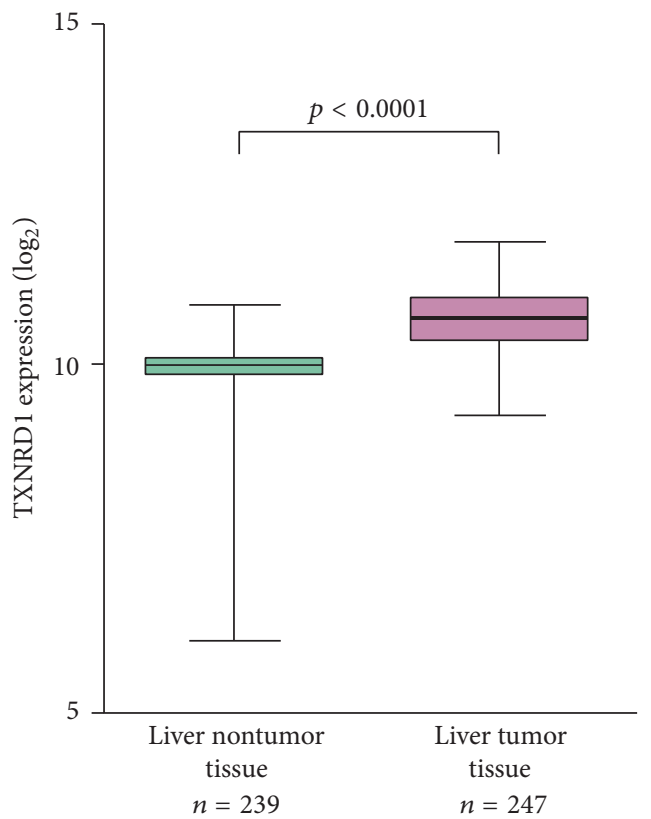

(a)

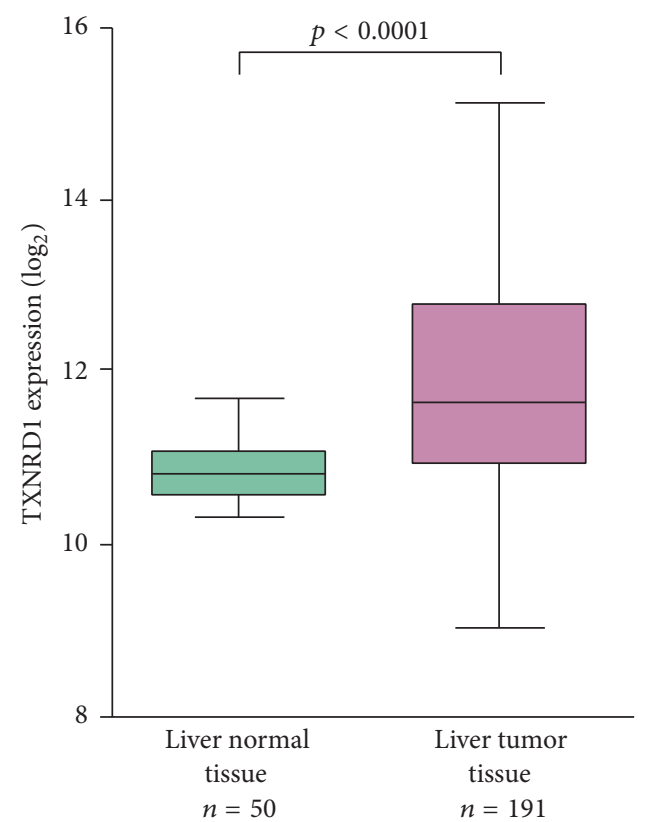

(b)

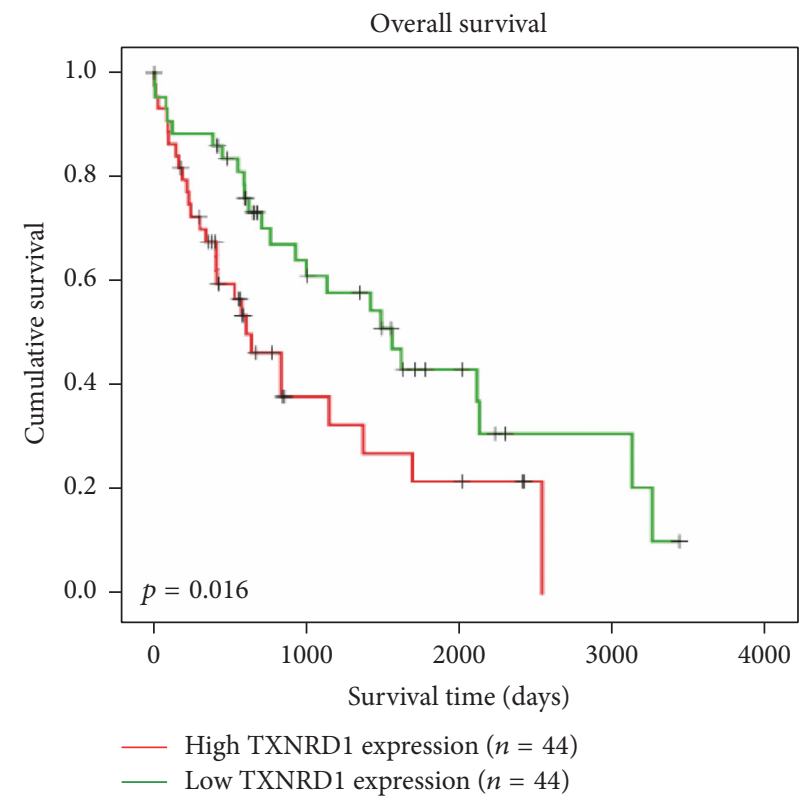

(c)

FIGURE 1: TXNRD1 was overexpressed in HCC tissues and patients with high TXNRD1 level had poor outcome. (a) The mRNA levels of TXNRD1 in HCC tissues and normal liver tissues, the data downloaded from GSE14520. (b) The mRNA levels of TXNRD1 in HCC tissues and normal liver tissues, the data downloaded from TCGA dataset. (c) Kaplan-Meier analysis for overall survival of HCC patients according to the level of TXNRD1. Data represent mean \pm SD.

upregulated in HCC tissues compared to normal live tissues $(p<0.0001)$ (Figure 1(b)). Kaplan-Meier survival curves suggested patients with high TXNRD1 have poor outcome $(p=0.016)$ (Figure 1(c)); this data also came from TCGA. Moreover, we determined TXNRD1 expression in HCC cells, and western blot assay suggested TXNRD1 protein level was upregulated in HCC cells compared to normal live cell LO2 (Figure 2(a)).
We further examined TXNRD1 protein level in 120 paraffin-embedded archived HCC tissues using IHC. IHC analysis suggested $113(94.2 \%)$ samples expressed TXNRD1 positively, and only 7 (5.8\%) samples expressed TXNRD1 negatively. 63 samples (52.5\%) had low TXNRD1 expression, 57 (47.5\%) had high TXNRD1 expression (Table 2), and TXNRD1 was mainly located in the cytoplasm. These samples included 30 stage I samples, 30 stage II samples, 


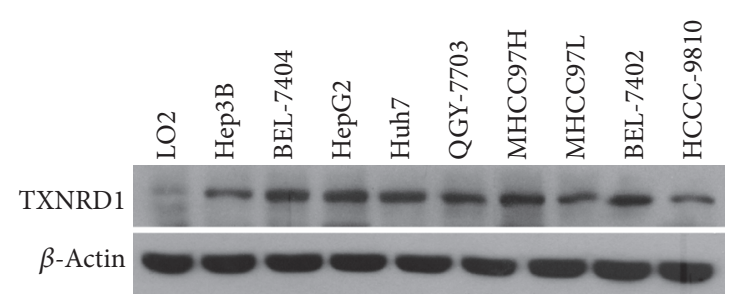

(a)

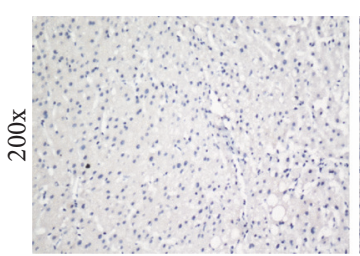

Normal liver

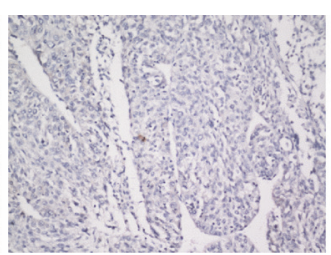

II

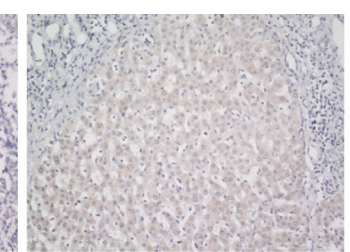

IIIA

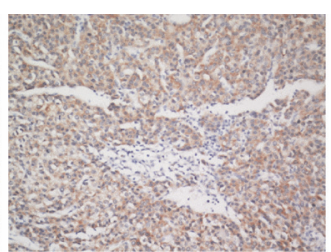

IIIB

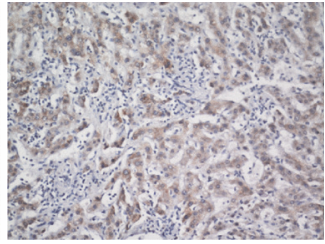

IV

Clinical stage

(b)

FIGURE 2: TXNRD1 expression was upregulated in HCC cells and was positively correlated with clinical stage. (a) Western blot analysis of TXNRD1 level in HCC cells and normal liver cell LO2. $\beta$-Actin was used as the loading control. (b) Representative photographs of TXNRD1 level in normal liver tissues, HCC samples in clinical stages II, IIIA, IIIB, and IV determined by IHC analysis. Original magnification: $\times 200$.

TABLE 2: The expression of TXNRD1 in HCC.

\begin{tabular}{lc}
\hline Expression of TXNRD1 & \\
\hline Negative & $7(5.8 \%)$ \\
Positive & $113(94.2 \%)$ \\
Low expression & $63(52.5 \%)$ \\
High expression & $57(47.5 \%)$ \\
\hline
\end{tabular}

34 stage III samples, and 26 stage IV samples. IHC assay also showed TXNRD1 level was positively correlated with advancing clinical stage (Figure 2(b)); the detailed data was shown as follows: $23 \%$ (7/30) for stage I, 37\% (11/30) for stage II, 53\% (18/34) for stage III, and 81\% (21/27) for stage IV (Table 3 ). This result suggested high TXNRD1 expression was associated with advancing clinical stage. These results suggested TXNRD1 was overexpressed in HCC tissues and might be a poor prognostic factor.

3.2. Relationship between Clinicopathologic Features and TXNRD1 Expression in HCC. We investigated the relationship between TXNRD1 level and clinicopathological characteristics of HCC patients and found that TXNRD1 level was significantly correlated with clinical stage $(p=3.5 e-5)$ and $\mathrm{N}$ classification ( $p=4.4 e-4$ using Chi-square test, $p=0.001$ using Fisher's exact test). TXNRD1 also was significantly correlated with $\mathrm{M}$ classification analyzed by Chisquare test $(p=0.037)$ but was not significantly correlated with $\mathrm{M}$ classification analyzed by Fisher's exact test $(p=$ 0.052). However, TXNRD1 level does not have significant correlation with gender, age, or T classification (Table 3). We used Spearman correlation analysis to confirm this result; Spearman correlation analysis showed TXNRD1 level was significantly correlated with clinical stage $(r=0.405, p=$ 5.3e - 8), $\mathrm{N}$ classification $(r=0.326, p=7.8 e-7)$, and $\mathrm{M}$ classification $(r=0.190, p=0.037)$, but TXNRD1 level was not significantly correlated with T classification $(r=0.178$, $p=0.052$ ) (Table 4). Taken together, high TXNRD1 level was positively correlated with clinical stage, $\mathrm{N}$ classification, and M classification.

3.3. Patients with High TXNRD1 Level Have Poor Outcome. Using Kaplan-Meier analysis and the log-rank test, we observed that patients with low TXNRD1 levels had longer overall survival time $(p<0.001$, Figure 3(a)). Subgroup analyses found that patients with high TXNRD1 levels had poor prognosis in the clinical classification I-II $(p<0.001)$ or in the clinical classification III-IV $(p<0.05)$, suggesting TXNRD1 also was an unfavorable prognostic factor for clinical classifications I-II and III-IV (Figures 3(a) and 3(b)). Univariate Cox-regression analysis showed both clinical stage and TXNRD1 level were the significant poor prognostic factors $(p=5.5 e-9$ and $p=8.5 e-8$, resp.), and multivariate Cox-regression analysis found clinical stage and TXNRD1 level were independent prognostic factor for HCC patients ( $p=6.1 e-7$ and $5.4 e-11$, resp.) (Table 5). These findings revealed that TXNRD1 was an independent prognostic factor for HCC patients; patients with high TXNRD1 level had shorter survival time than those who had low TXNRD1 level.

\section{Discussion}

In the present study, we investigated whether TXNRD1 could function as a prognostic factor for HCC patients. We first analyzed TXNRD1 expression using public database and found that TXNRD1 was significantly overexpressed in HCC tissues, and patients with high TXNRD1 level had poor outcome. Then, we further determined TXNRD1 expression in HCC 


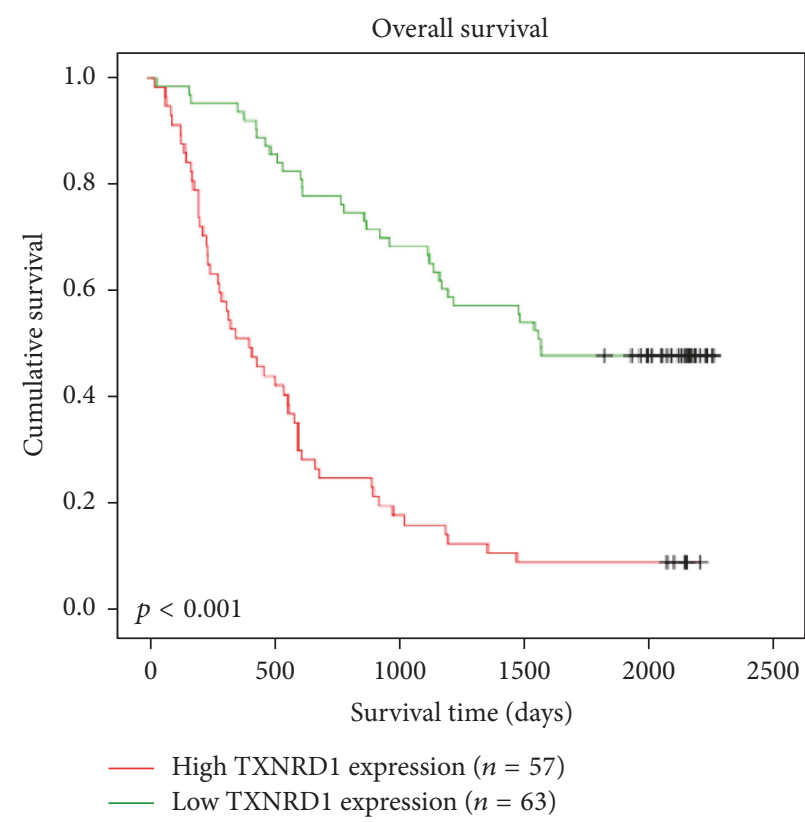

(a)

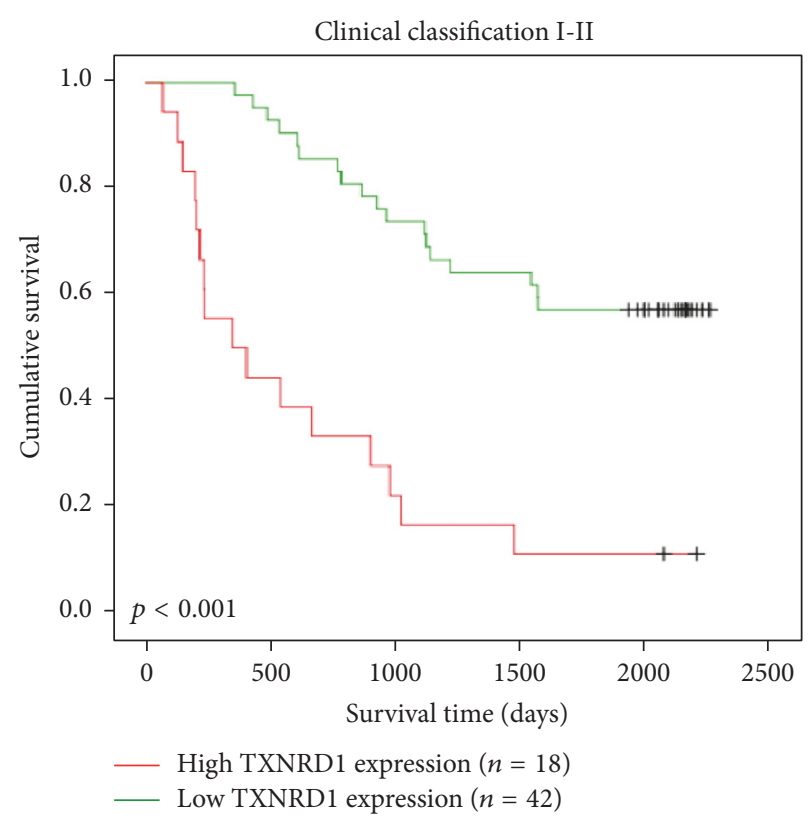

(b)

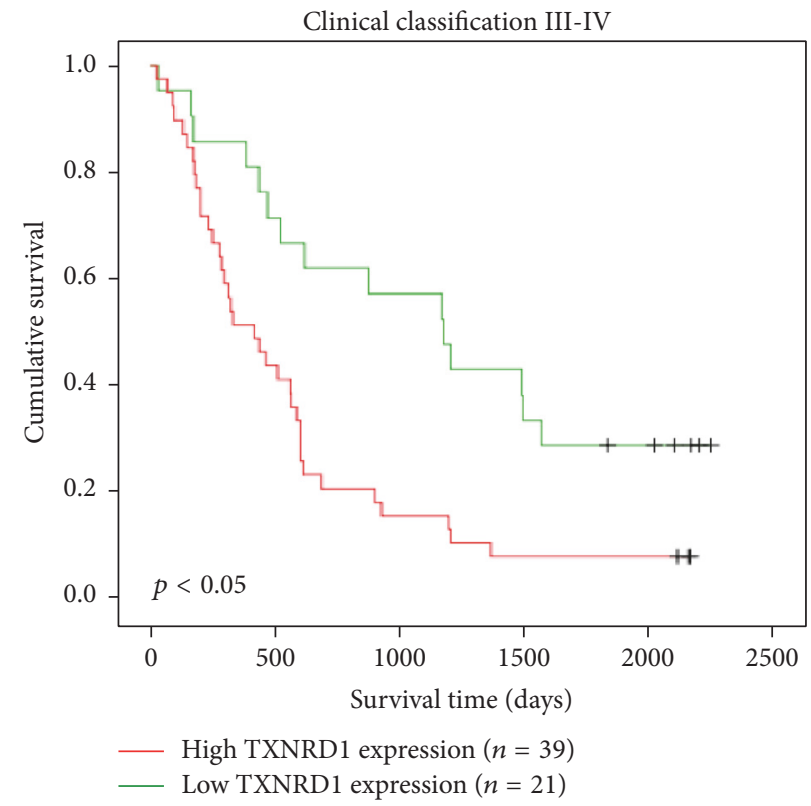

(c)

FIgURE 3: Kaplan-Meier analysis to investigate the prognostic value of TXNRD1 level in overall survival and different clinical stage. (a) All patients. (b) Patients with clinical classification I-II. (c) Patients with clinical classification III-IV.

cells and clinical specimens; TXNRD1 was overexpressed in HCC cells, and almost all collected specimens were TXNRD1positive. Statistical analyses revealed that high TXNRD1 expression was positively correlated with clinical stage, $\mathrm{N}$ classification, and $\mathrm{M}$ classification. The patients who had low TXNRD1 level had longer survival time. Multivariate analysis showed TXNRD1 was an independent prognostic biomarker for HCC patients.

Thioredoxin system comprised by thioredoxin reductase (TrxR/TXNDR), thioredoxin (Trx), and NADPH plays an important role in maintaining intracellular redox homeostasis. There are three TXNDRs: TXNRD1 is located in cytosol, TXNRD2 is located in mitochondria, and TXNRD3 is located in testis [14]. IHC analysis also found TXNRD1 was mainly presented in cytosol, and the role and molecular mechanisms of TXNRD1 in HCC progression will be explored further.

TXNRD1 is involved in protecting from ROS by itself and via its function together with TXN to serve as an electron donor for peroxiredoxins or ribonucleotide reductase involved in DNA replication and repair [14]. TXNRD1 has 
TABLE 3: Correlation between TXNRD1 expression and clinicopathological characteristics of HCC.

\begin{tabular}{|c|c|c|c|c|}
\hline \multirow{2}{*}{ Characteristics } & \multicolumn{2}{|c|}{ TXNRD1 } & \multirow{2}{*}{ Chi-square test $p$ value } & \multirow{2}{*}{ Fisher's exact test $p$ value } \\
\hline & Low number of or no cases & High number of cases & & \\
\hline \multicolumn{5}{|l|}{ Gender } \\
\hline Male & 44 & 44 & \multirow{2}{*}{0.363} & \multirow{2}{*}{0.412} \\
\hline Female & 19 & 13 & & \\
\hline \multicolumn{5}{|l|}{ Age (years) } \\
\hline$\geq 45$ & 40 & 36 & \multirow{2}{*}{0.970} & \multirow{2}{*}{1.000} \\
\hline$<45$ & 23 & 21 & & \\
\hline \multicolumn{5}{|l|}{ Clinical stage } \\
\hline I & 23 & 7 & \multirow{4}{*}{$3.5 e-5$} & \multirow{4}{*}{$3.5 e-5$} \\
\hline II & 19 & 11 & & \\
\hline III & 16 & 18 & & \\
\hline IV & 5 & 21 & & \\
\hline \multicolumn{5}{|l|}{ T classification } \\
\hline $\mathrm{T} 1$ & 24 & 15 & \multirow{4}{*}{0.265} & \multirow{4}{*}{0.263} \\
\hline $\mathrm{T} 2$ & 19 & 14 & & \\
\hline T3 & 8 & 10 & & \\
\hline $\mathrm{T} 4$ & 12 & 18 & & \\
\hline \multicolumn{5}{|l|}{$\mathrm{N}$ classification } \\
\hline N0 & 59 & 39 & \multirow{2}{*}{$4.4 e-4$} & \multirow{2}{*}{0.001} \\
\hline N1 & 4 & 18 & & \\
\hline \multicolumn{5}{|l|}{ M classification } \\
\hline No & 62 & 51 & \multirow{2}{*}{0.037} & \multirow{2}{*}{0.052} \\
\hline Yes & 1 & 6 & & \\
\hline
\end{tabular}

TABLE 4: Spearman correlation analysis between TXNRD1 and clinical pathologic factors.

\begin{tabular}{lcc}
\hline \multirow{2}{*}{ Variables } & \multicolumn{2}{c}{ TXNRD1 expression level } \\
& Spearman correlation & $p$ value \\
\hline Clinical stage & 0.405 & $5.3 e-8$ \\
T classification & 0.178 & 0.052 \\
N classification & 0.326 & $7.8 e-7$ \\
M classification & 0.190 & 0.037 \\
\hline
\end{tabular}

been demonstrated to be overexpressed in many cancers, and ROS level also increased in many tumors, including HCC [15]. TXNRD1 promotes tumor growth, DNA replication, and tumorigenicity [16, 17], and knockdown of TXNRD1 also increases sensitivity of cancer cells to some chemotherapy drugs $[18,19]$; these finding suggest it is a potential target for anticancer agents [20]. In HCC, ROS causes DNA damage and regulates p53, AP1, NF-AT, Nrf2/Maf, and multidrug resistance proteins to promote hepatocarcinogenesis and drug resistance [21]; we conferred that TXNRD1 might induce ROS generation to promote HCC development and drug resistance.

We found TXNRD1 could function as an unfavorable prognostic factor for HCC, while this finding is still to be replicated and to be verified in other patients' population. In summary, we found TXNRD1 was upregulated in HCC tissues and cells, and TXNRD1 level was increased with increasing clinical stage. High TXNRD1 level was positively correlated with clinical stage, $\mathrm{N}$ classification, and $\mathrm{M}$ classification, and patients with high TXNRD1 level had poor outcome; TXNRD1 was a novel biomarker for the prognosis for HCC patients.

\section{Ethical Approval}

All procedures performed in the studies involving human participants were in accordance with the ethical standards of the institution or practice at which the studies were conducted.

\section{Consent}

Informed consent was obtained from all the individual participants included in the study.

\section{Conflicts of Interest}

The authors declare that they have no conflicts of interest.

\section{Acknowledgments}

This work was supported by the State Key Projects on Infection Diseases of China (2012ZX10002017-005, 
TABLE 5: Univariate and multivariate analyses of various prognostic parameters in patients with HCC Cox-regression analysis.

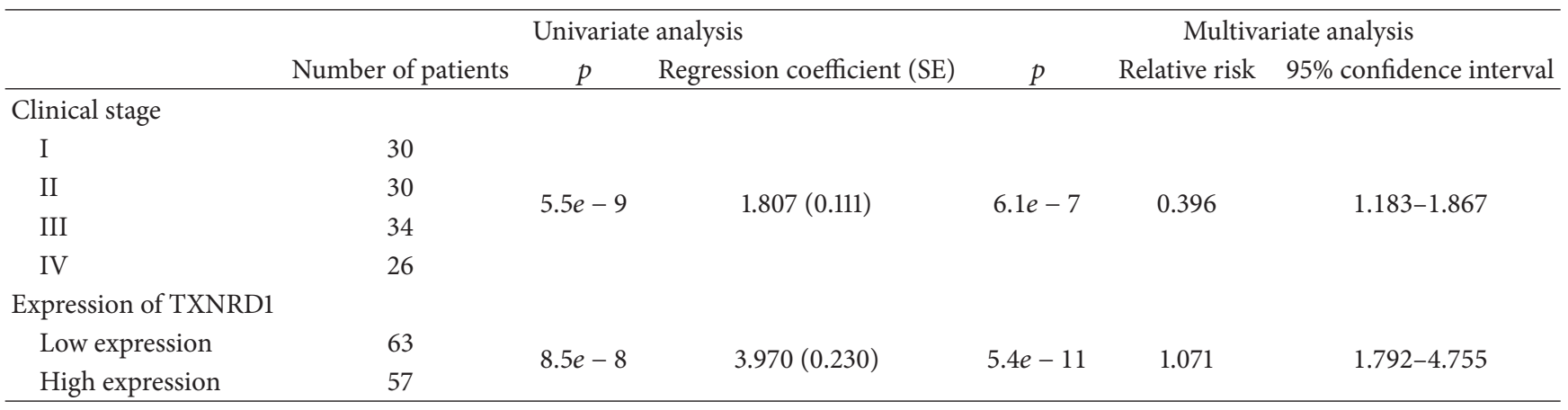

2012ZX10002016-023, and 2012ZX10002010-001-007), the National Natural Science Foundation of China (81372243, 81370575, and 81301331), Guangdong Natural Science Foundation (2014A030313131, 2015A030313756, 2015A030312013, and 2016A030313195), Science and Technology Planning Project of Guangdong Province (2014B020228003, 2014B030301041, and 2015B020226004), Science and Technology Planning Project of Guangzhou (201400000001-3, 158100076, and 2013J4100081), and Guangzhou Municipal Health Bureau (20141A011117, 20151A011112). These fundings were used to pay the salary of researchers and perform the experiments.

\section{References}

[1] L. A. Torre, F. Bray, R. L. Siegel, J. Ferlay, J. Lortet-Tieulent, and A. Jemal, "Global cancer statistics, 2012," CA Cancer Journal for Clinicians, vol. 65, no. 2, pp. 87-108, 2015.

[2] P. Nguyen, R. T. Awwad, D. D. K. Smart, D. R. Spitz, and D. Gius, "Thioredoxin reductase as a novel molecular target for cancer therapy," Cancer Letters, vol. 236, no. 2, pp. 164-174, 2006.

[3] E. S. J. Arnér and A. Holmgren, "Physiological functions of thioredoxin and thioredoxin reductase," European Journal of Biochemistry, vol. 267, no. 20, pp. 6102-6109, 2000.

[4] S. Iwasawa, Y. Yamano, Y. Takiguchi, H. Tanzawa, K. Tatsumi, and K. Uzawa, "Upregulation of thioredoxin reductase 1 in human oral squamous cell carcinoma," Oncology Reports, vol. 25, no. 3, pp. 637-644, 2011.

[5] A. P. Fernandes, A. Capitanio, M. Selenius, O. Brodin, A.-K. Rundlöf, and M. Björnstedt, "Expression profiles of thioredoxin family proteins in human lung cancer tissue: correlation with proliferation and differentiation," Histopathology, vol. 55, no. 3, pp. 313-320, 2009.

[6] Y. Soini, K. Kahlos, U. Näpänkangas et al., "Widespread expression of thioredoxin and thioredoxin reductase in non-small cell lung carcinoma," Clinical Cancer Research, vol. 7, no. 6, pp. 17501757, 2001.

[7] A. Cañas, L. M. López-Sánchez, A. Valverde-Estepa et al., "Maintenance of S-nitrosothiol homeostasis plays an important role in growth suppression of estrogen receptor-positive breast tumors," Breast Cancer Research, vol. 14, no. 6, article R153, 2012.

[8] C. Cadenas, D. Franckenstein, M. Schmidt et al., "Role of thioredoxin reductase 1 and thioredoxin interacting protein in prognosis of breast cancer," Breast Cancer Research, vol. 12, no. 3, article R44, 2010.
[9] H. Esen, F. Erdi, B. Kaya, B. Feyzioglu, F. Keskin, and L. S. Demir, "Tissue thioredoxin reductase-1 expression in astrocytomas of different grades," Journal of Neuro-Oncology, vol. 121, no. 3, pp. 451-458, 2015.

[10] B. A. Carlson, M.-H. Yoo, R. Tobe et al., "Thioredoxin reductase 1 protects against chemically induced hepatocarcinogenesis via control of cellular redox homeostasis," Carcinogenesis, vol. 33, no. 9, pp. 1806-1813, 2012.

[11] D. Duan, B. Zhang, J. Yao et al., "Gambogic acid induces apoptosis in hepatocellular carcinoma SMMC-7721 cells by targeting cytosolic thioredoxin reductase," Free Radical Biology and Medicine, vol. 69, pp. 15-25, 2014.

[12] L. Chen, Y. Li, C. H. Lin et al., "Recoding RNA editing of AZIN1 predisposes to hepatocellular carcinoma," Nature Medicine, vol. 19, no. 2, pp. 209-216, 2013.

[13] G. Lu, T. Shan, S. He et al., "Overexpression of CENP-H as a novel prognostic biomarker for human hepatocellular carcinoma progression and patient survival," Oncology Reports, vol. 30, no. 5, pp. 2238-2244, 2013.

[14] A. Holmgren and J. Lu, "Thioredoxin and thioredoxin reductase: current research with special reference to human disease," Biochemical and Biophysical Research Communications, vol. 396, no. 1, pp. 120-124, 2010.

[15] D. Trachootham, J. Alexandre, and P. Huang, "Targeting cancer cells by ROS-mediated mechanisms: a radical therapeutic approach?" Nature Reviews Drug Discovery, vol. 8, no. 7, pp. 579-591, 2009.

[16] M.-H. Yoo, X.-M. Xu, B. A. Carlson, V. N. Gladyshev, and D. L. Hatfield, "Thioredoxin reductase 1 deficiency reverses tumor phenotype and tumorigenicity of lung carcinoma cells," Journal of Biological Chemistry, vol. 281, no. 19, pp. 13005-13008, 2006.

[17] M.-H. Yoo, X.-M. Xu, B. A. Carlson, A. D. Patterson, V. N. Gladyshev, and D. L. Hatfield, "Targeting thioredoxin reductase 1 reduction in cancer cells inhibits self-sufficient growth and DNA replication," PLoS ONE, vol. 2, no. 10, Article ID el112, 2007.

[18] R. L. Poerschke and P. J. Moos, “Thioredoxin reductase 1 knockdown enhances selenazolidine cytotoxicity in human lung cancer cells via mitochondrial dysfunction," Biochemical Pharmacology, vol. 81, no. 2, pp. 211-221, 2011.

[19] R. Tobe, M.-H. Yoo, N. Fradejas et al., “Thioredoxin reductase 1 deficiency enhances selenite toxicity in cancer cells via a thioredoxin-independent mechanism," Biochemical Journal, vol. 445, no. 3, pp. 423-430, 2012. 
[20] D. K. Smart, K. L. Ortiz, D. Mattson et al., “Thioredoxin reductase as a potential molecular target for anticancer agents that induce oxidative stress," Cancer Research, vol. 64, no. 18, pp. 6716-6724, 2004.

[21] M. T. Kuo and N. Savaraj, "Roles of reactive oxygen species in hepatocarcinogenesis and drug resistance gene expression in liver cancers," Molecular Carcinogenesis, vol. 45, no. 9, pp. 701709, 2006. 


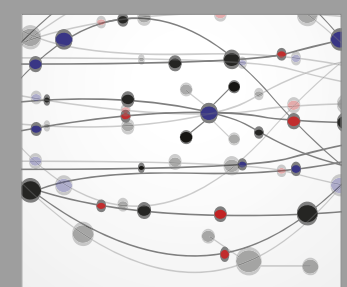

The Scientific World Journal
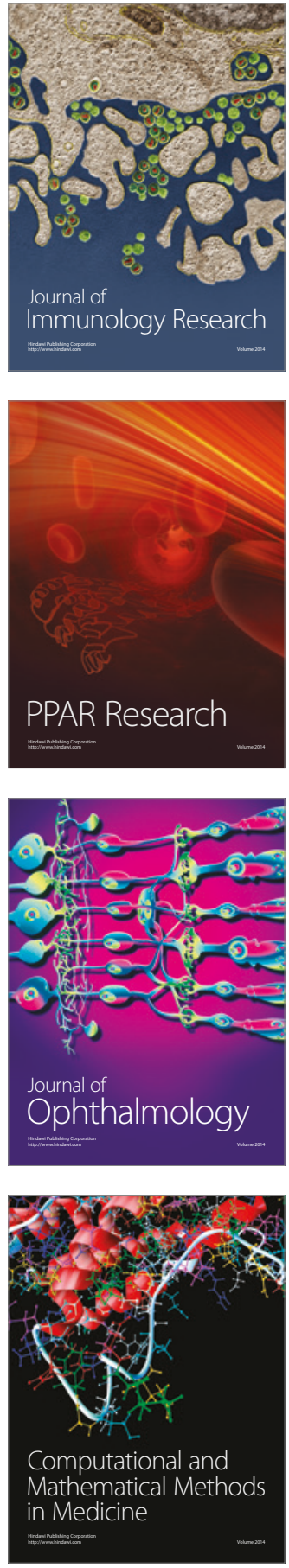

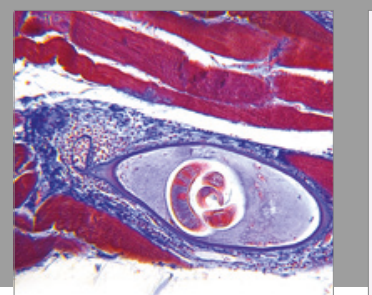

Gastroenterology Research and Practice
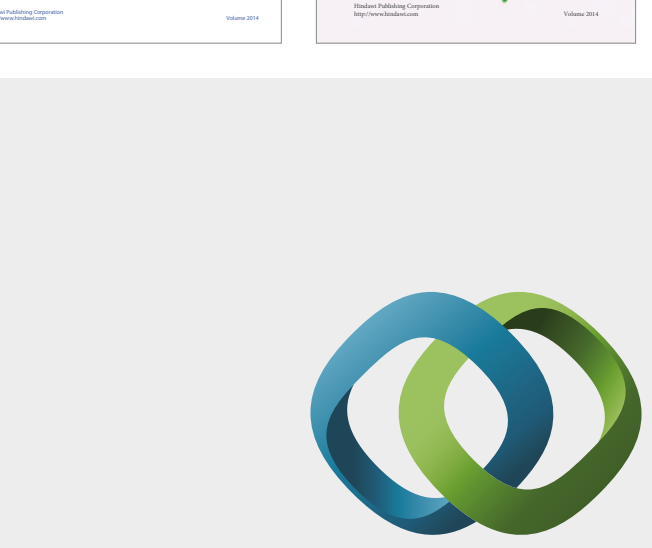

\section{Hindawi}

Submit your manuscripts at

https://www.hindawi.com
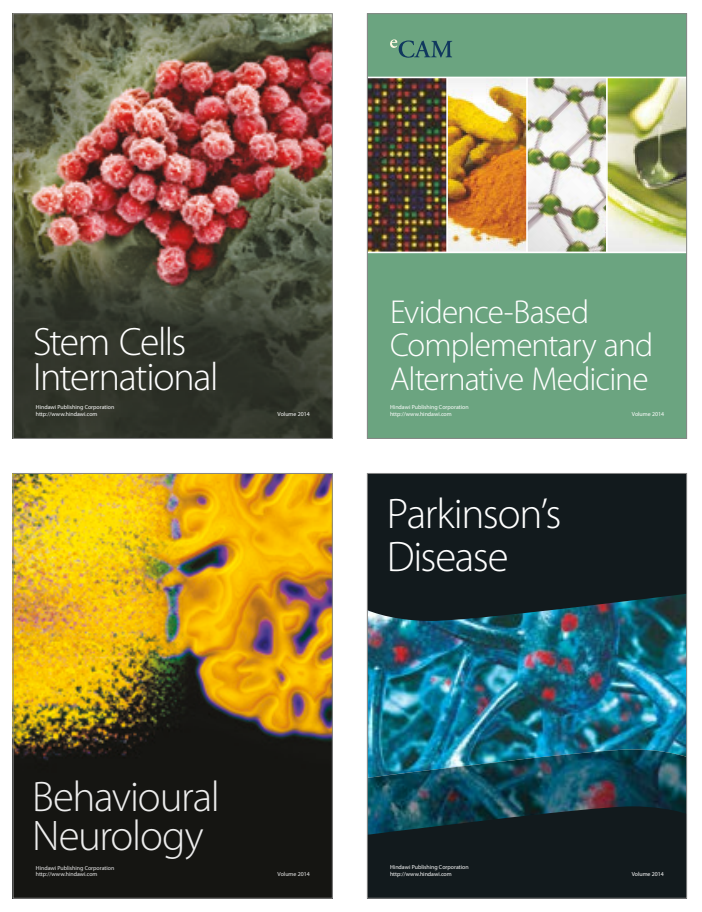
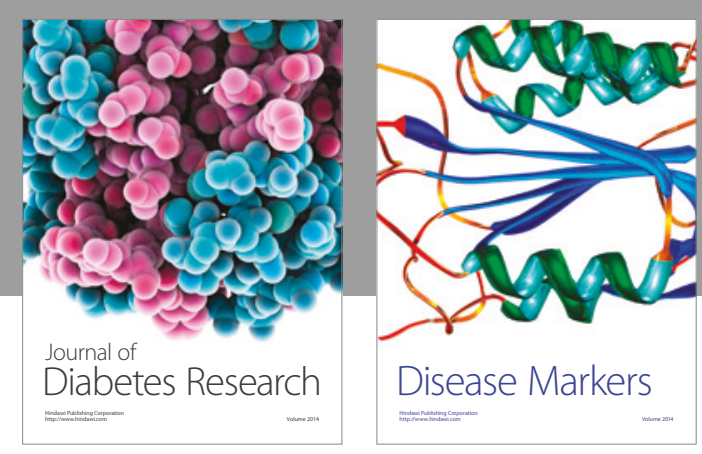

Disease Markers
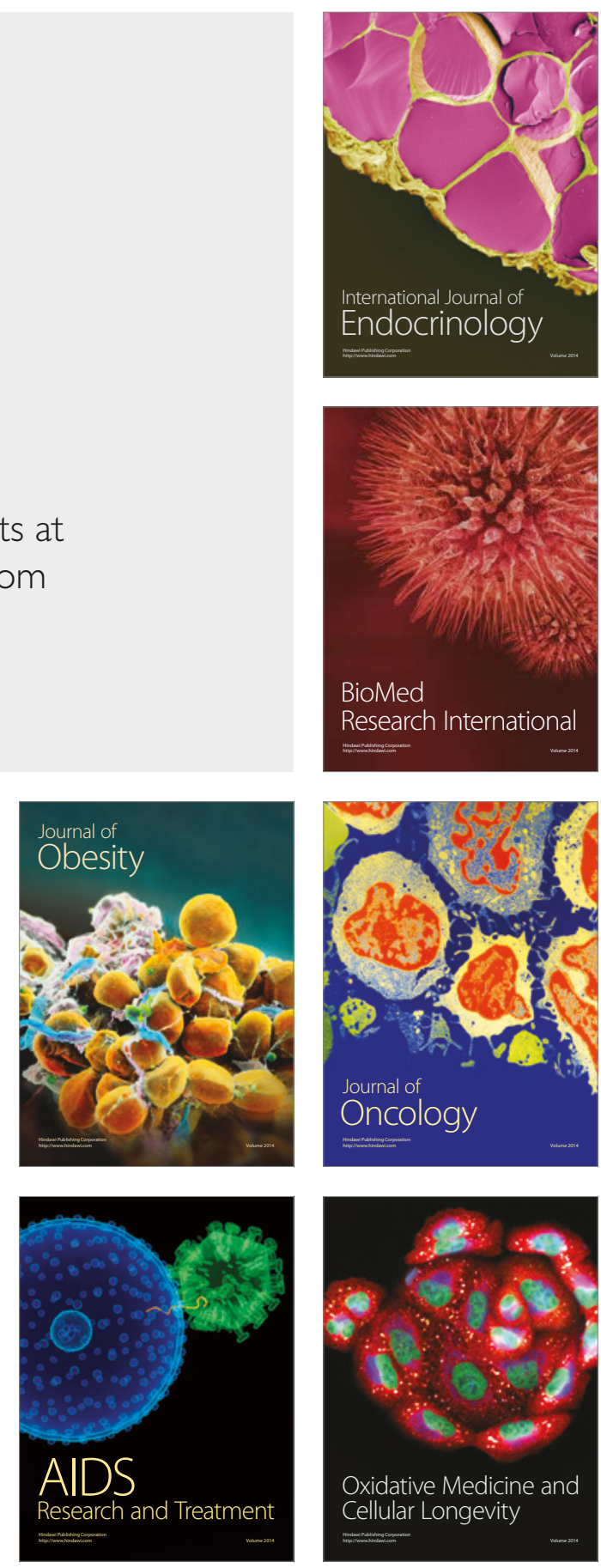